MATEC Web of Conferences 22,01018 (2015)

DOI: $10.1051 /$ matec conf/ 20152201018

(C) Owned by the authors, published by EDP Sciences, 2015

\title{
Research of Digital Interface Layout Design based on Eye-tracking
}

\author{
Jiang Shao, Chengqi Xue*, Fang Wang, Haiyan Wang, Wencheng Tang \& Mo Chen \\ School of Mechanical Engineering, Southeast University, Nanjing, Jiangsu, China \\ Mingwu Kang \\ Science and Technology on Electro-optic Control Laboratory, Luoyang, Henan, China
}

\begin{abstract}
The aim of this paper is to improve the low service efficiency and unsmooth human-computer interaction caused by currently irrational layouts of digital interfaces for complex systems. Also, three common layout structures for digital interfaces are to be presented and five layout types appropriate for multilevel digital interfaces are to be summarized. Based on the eye tracking technology, an assessment was conducted in advantages and disadvantages of different layout types through subjects' search efficiency. Based on data and results, this study constructed a matching model which is appropriate for multilevel digital interface layout and verified the fact that the task element is a significant and important aspect of layout design. A scientific experimental model of research on digital interfaces for complex systems is provided. Both data and conclusions of the eye movement experiment provide a reference for layout designs of interfaces for complex systems with different task characteristics.
\end{abstract}

Keywords: eye-tracking experiment; layout of interface; human computer interaction interface

\section{INTRODUCTION}

With the wide application of digital interfaces in complex systems, it has become increasingly urgent to design interfaces for complex systems. Interface layout is an integral part of the digital interface, and a good one will be helpful for users to acquire interface information rapidly and efficiently, carry out visual search and succeed in related operations, thereby strengthening users' positive cognition of the system.

Interface layout refers to an approach for rational layout of interface elements within a limited range, by which a messy interface and farraginous contents will be induced according to the need of the general layout, so as to carry out the interrelated organization and arrangement and hash out the relationship between interface elements and space, providing a smooth user experience for the user [1,2]. The interface layout design is a process of multivariate coordination design and continuous iteration-feedback. Many specialists and scholars at home and abroad have conducted research on the web interface layout to improve user's interactive experience in browsing the web. Altaboli used correlation analysis to analyze subjective and objective measures in visual design of the web interface [3]. Singh investigated AGA-based approach to improve web page aesthetics [4]. Teng proposed three kinds of interfaces in the application context of the process plant based on the FBS methodology and the PCP [5]. Wang indicated that stacked layout of the visual items allowed users to find the intended targets rapidly and form a direct and rapid approach to search route from the perspective of cognition [6]. Zhao ap*Corresponding author: ipd xcq@,seu.edu.cn plied the eye tracking technology to investigate differences between visual search efficiency and subjective satisfaction from various webpage layouts by analyzing webpage layout factors [7]. Abovementioned scholars proposed some arguments, but no system partitioning and no further research has been made on layout types of interfaces for complex systems. However, their theories also suggest the significance in research on layouts of interfaces for complex systems.

\section{EYE TRACKING TECHNOLOGY}

The eye tracking technology can be used for investigation and assessment of digital interface layouts because it reveals spatial positions where users would pay attention to or be interested to subjects as well as the process of attention shift by tracking eye movement and pupil change. The eye tracking technology is more direct and efficient compared with traditional approaches. Research on physiological properties of eye movement can help us understand and find out how users acquire information from the interface and what the rule is. The line of sight in human is characterized by linearity, naturalness and bidirectionality [8]. In the process of cognition, visual fixation is not merely an inherent physiological property, but also closely correlated with cognitive activities of the brain. When the brain is working, eyes will gaze. The gaze time of the eye increases as the think time extends. Therefore, the time when users' eyes fixated on the screen may roughly show how much time their cogni- 
tive activities need when they use digital interfaces [9].

So far, the eye tracking technology is widely used in usability research, psychology, ergonomics, clinical research, aviation and transportation. For example, Cheng presented an approach to the eye tracking for mobile device based on human-machine interaction [10]. Jin presented a vehicle human-computer interface layout design reasoning system and realized tight integration of this system with the 3D CAD platform [11]. Wang proposed an interface evaluation method of fighter-driving display-control system based on the eye tracking technology [12]. Weinreich carried out an empirical study of enterprise web and search engine interface [13]. This shows that the eye tracking technology plays a significant role in research of human-computer interface. However, few researches have focused on layouts of interfaces for complex systems.

\section{CLASSIFICATION OF MULTILEVEL INTER- FACE LAYOUTS}

The position relationship of interface elements results in digital interface layout. An array of combination modes of multiple interface elements leads to different interface types. The reason why users use digital interfaces for complex systems is to fulfill specific performance tasks. The task complexity is considered as the most important factor influencing information access $[14,15]$. Such complexity can be defined from different perspectives. Campbell described task complexity as three typologies: (a) a primarily psychological experience, (b) an interaction between task and person characteristics, and (c) a function of objective task characteristics [16]. Navigation elements play a crucial role in guiding users to fulfill task operations; however, there are differences in layout type among different types of digital interfaces. Starting with the structures and positions of navigation elements and combining with common layout types of digital interfaces for complex systems, a matching relationship is constructed in various types of digital interfaces and layouts. For digital interface for single-level task, there are three common layout types: Types A, B (B1 and $\mathrm{B} 2)$, and $\mathrm{C}(\mathrm{C} 1$ and $\mathrm{C} 2)$. As shown in Figure 1, the shadow area represents the position of a navigation element.

An interface for complex system tends to include multilevel digital interfaces due to large amounts of information. For such interfaces, layout changes caused by structures and positions of navigation elements become more complex, thus there will be more layout types, which evolve from five basic types into a total of 34 types. The layout type A is shown in Figure 2 , with a total of 10 subtypes, in which numbers represent a high-to-low navigation element hierarchy. Various layout types are numbered to contribute to experimental data analysis.

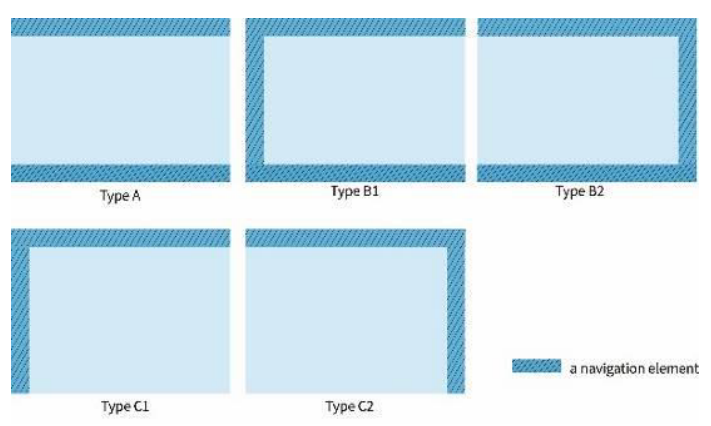

Figure 1. Layout types of single-level digital interfaces

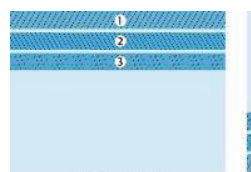

TypeA No.1
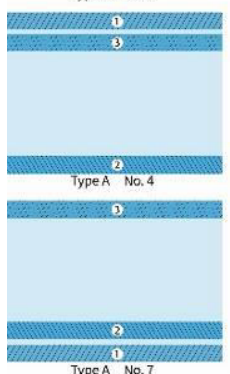

Iype A No.7
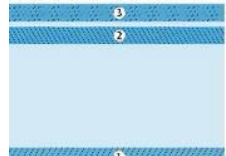

Type A No. 10

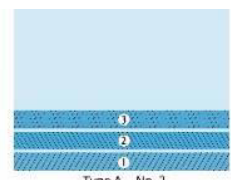

Type A No.2
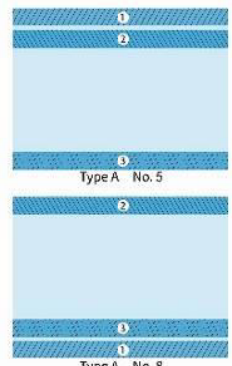

Type A No. 8

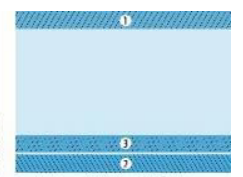

Type A No.3
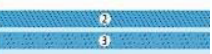

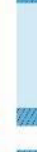
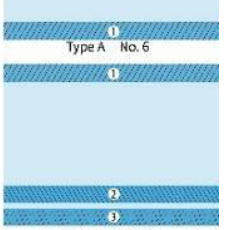

Figure 2. Layout Type A of multilevel digital interface

Other layouts, Types B1, B2, C1 and C2, have six variations respectively, as shown in Figure 3, Figure 4, Figure 5, and Figure 6:
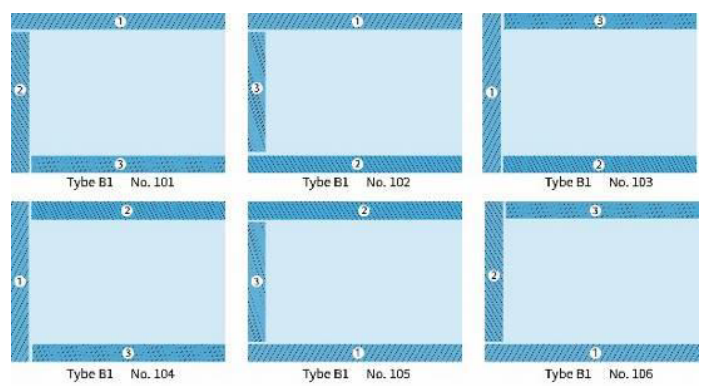

Figure 3. Layout Type B1 of multilevel digital interface 

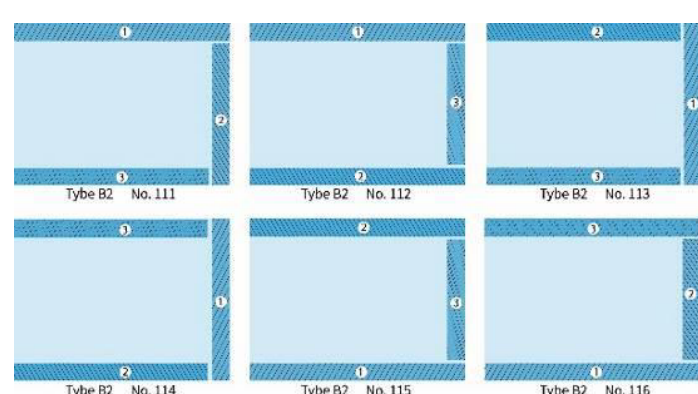

Figure 4. Layout Type B2 of multilevel digital interface

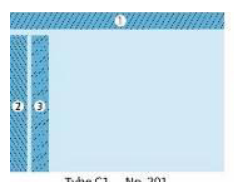

Tybe C1 No. 201

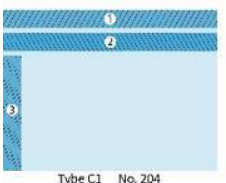

Tybe C1 No. 204

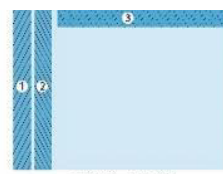

TybeC1 No. 202

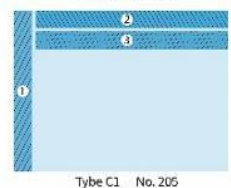

Tybe C1 No. 205

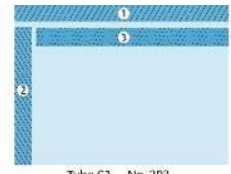

Tybe C1 No.203

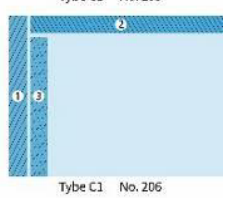

Figure 5. Layout Type C1 of multilevel digital interface
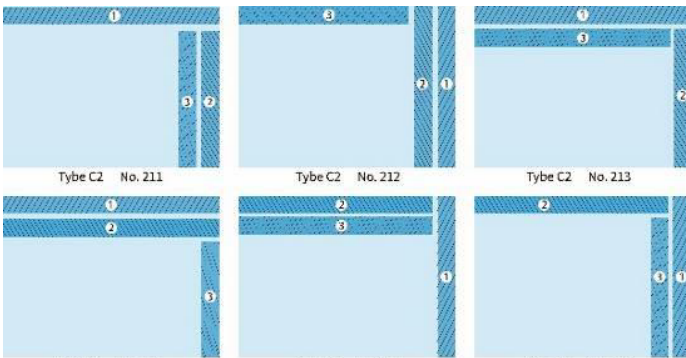

Tybe C2 No.215

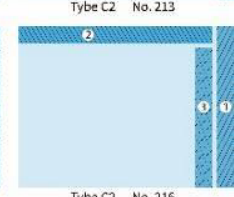

Figure 6. Layout Type C2 of multilevel digital interface

\section{EXPERIMENTAL DESIGN}

\subsection{Objective}

Studies by Farzan, Kammerer showed a significant difference in visual search efficiency between subjects in pages with different layouts $[17,18]$. Based on the classification and generalization of layouts of digital interfaces for complex systems, the eye tracking technology was used to test the interface layout types; influence of position of interface element on interface layout was examined; advantages and disadvantages of different interface layouts were assessed by determining users' total fixation duration, numbers of fixation points and scanning paths when users executed the same task in different layouts.

\subsection{Subjects}

The information monitoring system administrators were experimental tasks' object user group. In order to be familiar with the system compared with administrators, subjects were required to learn the experiment task previously. There were a total of 30 subjects (6 doctoral students and 24 postgraduates aged 20 to 30 years with male-female ratio of 2:3) with normal vision or corrected visual acuity in this experiment.

\subsection{Experimental procedures}

Interfaces for experimental tasks were based on the complex information monitoring system. As shown in Figure 7 , subjects were required to find following elements orderly in continuous presented interfaces: Monitoring Center, Monitoring Center Management, Application Monitoring and Application Ranking. Each subject had to press the space bar to respond to each element found, while the interface fed it back until the task termination after "Application Ranking" was found. Experiment was conducted on subjects in a randomly presented 34 (basic layout type) $\times 1$ manner. A common digital resolution of $1024 \times 768$ was used in interfaces designed in this experiment. Interfaces were grayed in order to avoid interference from other factors.

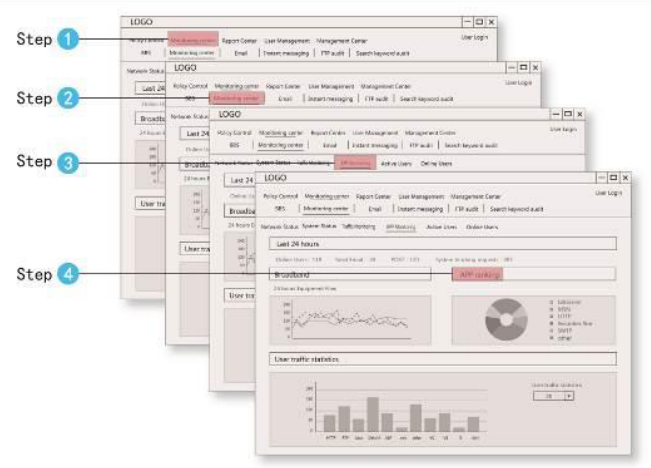

Figure 7. Schematic diagram of application interface for experimental task

\section{EXPERIMENTAL DATA ANALYSIS}

\subsection{Analysis of total number of fixation points on the interface}

There were 30 sets of subjective data in the eye tracking experiment, and four sets of data were invalid due to the insufficient sampling rate, whereas 26 sets were valid. To analyze and compare them easily, 34 layout types were coded as follows: 10 Types of layout A are respectively numbered 1 to $10 ; 6$ Types of layout B1 are respectively numbered 101 to 106; 6 Types of layout B2 are respectively numbered 111 to $116 ; 6$ 
Types of layout $\mathrm{C} 1$ are respectively numbered 201 to 206; and 6 Types of layout C2 are respectively numbered 211 to 216 .

\subsubsection{Statistical analysis among five layout types} Descriptive statistical analysis of total numbers of fixation points was listed in Table 1:

Table 1. Descriptive statistical analysis of total number of fixation points of among five interface types

\begin{tabular}{llll}
\hline Layout type & Sample size & Mean & SD \\
Type A & 260 & 21.12 & 8.376 \\
Type B1 & 156 & 20.38 & 7.703 \\
Type B2 & 156 & 21.36 & 8.637 \\
Type C1 & 156 & 18.26 & 6.946 \\
Type C2 & 156 & 18.73 & 7.365 \\
Total & 884 & 20.11 & 7.967 \\
\hline
\end{tabular}

Test for homogeneity of variance was conducted in the total number of fixation points using Levene's test, with a probability of 0.077 larger than the significance level of 0.05 , which satisfied the prerequisite for analysis of variance (ANOVA). One-way ANOVA was conducted on these five sets of data. It is supposed that different types of interface layout have no significant effects on the number of fixation points, and the significance level was 0.05 . The corresponding Results showed that the null hypothesis was rejected if $\mathrm{F}=5.643$ and $\mathrm{P}<0.05$. Thus, in the experimental task, different types of interface layout had significant effects on the number of fixation points. LSD was used to check the multiple comparison results among layouts. Data showing significant differences were listed in Table 2:

Table 2. Multiple comparison analysis of total numbers of fixation points among five interface layout types

\begin{tabular}{llll}
\hline (I) & (J) & Mean difference (I-J) & Significance \\
\hline \multirow{2}{*}{$\mathrm{A}$} & $\mathrm{C} 1$ & $2.863^{*}$ & 0.000 \\
\cline { 2 - 4 } & $\mathrm{C} 2$ & $2.394^{*}$ & 0.002 \\
\hline \multirow{2}{*}{$\mathrm{B} 1$} & $\mathrm{C} 1$ & $2.123^{*}$ & 0.016 \\
\hline \multirow{2}{*}{$\mathrm{B} 2$} & $\mathrm{C} 1$ & $3.105^{*}$ & 0.000 \\
\cline { 2 - 4 } & $\mathrm{C} 2$ & $2.636^{*}$ & 0.003 \\
\hline & $\mathrm{A}$ & $-2.863^{*}$ & 0.000 \\
\cline { 2 - 4 } $\mathrm{C} 1$ & $\mathrm{~B} 1$ & $-2.123^{*}$ & 0.016 \\
\cline { 2 - 4 } & $\mathrm{B} 2$ & $-3.105^{*}$ & 0.000 \\
\hline \multirow{2}{*}{$\mathrm{C} 2$} & $\mathrm{~A}$ & $-2.394^{*}$ & 0.002 \\
\cline { 2 - 4 } & B2 & $-2.636^{*}$ & 0.003 \\
\hline
\end{tabular}

*. The mean difference is significant at the 0.05 level.

It could be concluded that: a) The mean total numbers of fixation points of layout Type $\mathrm{C} 1$ was significantly less than those of other layout types with a significant difference;

b) The mean total numbers of fixation points of layout Type $\mathrm{C} 2$ was significantly less than those of layouts Types A and B2 with a significant difference;

c) There was no significant difference in mean total numbers of fixation points between layouts Types $\mathrm{C} 1$ and $\mathrm{C} 2$;

d) There was no significant difference between layouts Type A and Type B; however, the average total numbers of fixation points of the former were relatively more.

\subsubsection{Statistical analysis among 34 layout types}

There were a total of 34 types of interface layout in the experimental task. Descriptive statistical analysis of total numbers of fixation points was listed in Table 3:

Table 3. Descriptive statistical analysis of mean total numbers of fixation points of 34 interface layout types

\begin{tabular}{lllllllll} 
A & Mean & SD & B & Mean & SD & C & Mean & SD \\
\hline 1 & 20.12 & 7.388 & 101 & 18.58 & 5.805 & 201 & 18.15 & 4.066 \\
\hline 2 & 21.31 & 5.129 & 102 & 20.73 & 7.368 & 202 & 18.08 & 7.451 \\
\hline 3 & 20.00 & 7.288 & 103 & 19.38 & 5.441 & 203 & 21.31 & 6.565 \\
\hline 4 & 25.19 & 7.392 & 104 & 22.62 & 8.949 & 204 & 18.38 & 7.419 \\
\hline 5 & 18.54 & 5.630 & 105 & 22.04 & 5.737 & 205 & 17.58 & 3.797 \\
\hline 6 & 24.08 & 9.679 & 106 & 23.65 & 5.306 & 206 & 20.27 & 5.625 \\
\hline 7 & 25.50 & 9.162 & 111 & 18.73 & 7.948 & 211 & 17.31 & 4.541 \\
\hline 8 & 21.65 & 6.480 & 112 & 19.38 & 6.456 & 212 & .19 .85 & 7.983 \\
\hline 9 & 17.42 & 4.933 & 113 & 21.69 & 8.960 & 213 & 22.50 & 9.880 \\
\hline 10 & 24.50 & 6.825 & 114 & 24.81 & 7.985 & 214 & 18.23 & 4.761 \\
\hline & & & 115 & 21.85 & 5.576 & 215 & 18.35 & 4.454 \\
\hline & & & 116 & 26.38 & 6.357 & 216 & 21.23 & 4.555 \\
\hline Total & & & & & & & & \\
\end{tabular}

Test for homogeneity of variance was conducted in these data using Levene's test with a probability of 0.052 larger than a significance level of 0.05 , which satisfied the prerequisite for ANOVA. One-way ANOVA was conducted on these 34 sets of data. It is suppose that different types of interface layout have no significant effects on the number of fixation points, and the significance level was 0.05 . The corresponding Results showed that the null hypothesis was rejected if $\mathrm{F}=3.801$ and $\mathrm{P}<0.05$. Thus, in the experimental task, different types of interface layout had significant effects on the number of fixation points.

In conclusion, among layouts Type A, total mean numbers of fixation points of layouts $1,3,5$ and 9 
were significantly less than those of layouts $4,6,7$ and 10 with a significant difference; among layouts B1, total mean numbers of fixation points of layouts 101 and 103 were significantly less than that of layout 106 with a significant difference; among layouts Type B2, average total numbers of points of fixation of layouts 111 and 112 were significantly less than those of layouts 114 and 116 with a significant difference; among layouts Type $\mathrm{C} 1$, the average total number of fixation points of layout 205 was significantly less than that of layout 203 with a significant difference; among layouts Type $\mathrm{C} 2$, the average total number of fixation points of layout 211 was significantly less than those of layouts 213 and 215 with a significant difference.

\subsection{Analysis of the total fixation duration of the in terface}

5.2.1 Statistical analysis among five layout types Descriptive statistical analysis of total fixation duration was listed in Table 4:

Table 4. Descriptive statistical analysis of average total fixation durations of five interface layout types

\begin{tabular}{llll}
\hline Layout type & Sample size & Mean & SD \\
\hline $\mathrm{A}$ & 260 & 4.7151 & 2.23110 \\
\hline $\mathrm{B} 1$ & 156 & 4.4544 & 1.67306 \\
\hline $\mathrm{B} 2$ & 156 & 4.7230 & 2.28115 \\
\hline $\mathrm{C} 1$ & 156 & 3.9981 & 1.81982 \\
\hline $\mathrm{C} 2$ & 156 & 4.0945 & 1.60339 \\
\hline Total & 884 & 4.4344 & 1.99762 \\
\hline
\end{tabular}

Test for homogeneity of variance was conducted in total fixation duration by using Levene's test, with a probability of 0.0313 less than a significance level of 0.05 , which did not satisfy the prerequisite for ANOVA. One-way ANOVA was conducted on these five sets of data. Dunnett's T3 test was used to check multiple comparison results among layouts. Data showing a significant difference were listed in Table 5:
Table 5.Multiple comparison analysis of average total fixation durations among five interface layout types

\begin{tabular}{llll}
\hline (I) & (J) & Mean difference(I-J) & Significance \\
\hline \multirow{2}{*}{$\mathrm{A}$} & $\mathrm{C} 1$ & $0.71702^{*}$ & 0.000 \\
\cline { 2 - 4 } & $\mathrm{C} 2$ & $0.62060^{*}$ & 0.002 \\
\hline $\mathrm{B} 1$ & $\mathrm{C} 1$ & $0.45630^{*}$ & 0.038 \\
\hline \multirow{2}{*}{ B2 } & $\mathrm{C} 1$ & $0.72494^{*}$ & 0.001 \\
\cline { 2 - 4 } & $\mathrm{C} 2$ & $0.62852^{*}$ & 0.004 \\
\hline \multirow{2}{*}{$\mathrm{C} 1$} & $\mathrm{~A}$ & $-0.71702^{*}$ & 0.000 \\
\cline { 2 - 4 } & $\mathrm{B} 1$ & $-0.45630^{*}$ & 0.038 \\
\cline { 2 - 4 } & B2 & $-0.72494^{*}$ & 0.001 \\
\hline \multirow{2}{*}{$\mathrm{C} 2$} & $\mathrm{~A}$ & $-0.62060^{*}$ & 0.002 \\
\cline { 2 - 4 } & B2 & $-0.62852^{*}$ & 0.004 \\
\hline
\end{tabular}

*. The mean difference is significant at the 0.05 level.

It can be concluded that:

a) The mean total fixation duration of layout Type C1 was significantly less than those of other layout types, with a significant difference;

b) The mean total fixation duration of layout Type C2 was significantly less than those of layouts Types A and B2 with a significant difference;

c) There was no significant difference in mean total fixation duration between layouts Types $\mathrm{C} 1$ and $\mathrm{C} 2$;

d) There was no significant difference between layouts Type A and Type B. Meanwhile, it can be seen that these results were similar to those of total mean numbers of points of fixation of five layout types.

\subsubsection{Statistical analysis among 34 layout types}

Statistical analysis of total fixation durations of 34 layout types was listed in Table 6:

Table 6. Descriptive statistical analysis of mean total fixation durations of 34 interface layout types

\begin{tabular}{llllllllll}
\hline Type A & Mean & SD & Type B & Mean & SD & Type C & Mean & SD \\
\hline 1 & 4.4446 & 1.81625 & 101 & 4.1296 & 1.13658 & 201 & 4.0392 & 1.30303 \\
\hline 2 & 4.7131 & 1.11145 & 102 & 4.4508 & 1.42180 & 202 & 3.8842 & 2.14081 \\
\hline 3 & 4.5608 & 2.00096 & 103 & 4.2292 & 1.60572 & 203 & 4.5115 & 1.75432 \\
\hline 4 & 5.3554 & 1.62827 & 104 & 4.8938 & 1.70423 & 204 & 4.0173 & 1.81249 \\
\hline 5 & 4.1192 & 1.30434 & 105 & 4.8896 & 0.89745 & 205 & 3.8238 & 1.14841 \\
\hline 6 & 5.2573 & 2.33527 & 106 & 5.1612 & 1.60719 & 206 & 4.6350 & 1.66896 \\
\hline
\end{tabular}


MATEC Web of Conferences

\begin{tabular}{lllllllll}
\hline 7 & & & & & & & \\
\hline 8.7419 & 2.27290 & 111 & 3.8996 & 1.66714 & 211 & 3.6292 & 1.01909 \\
\hline 9 & 4.5842 & 1.83934 & 112 & 4.4219 & 2.12434 & 212 & .4 .3500 & 1.63325 \\
\hline 10 & 3.8773 & 1.06902 & 113 & 4.8500 & 1.91225 & 213 & 4.8231 & 1.80899 \\
\hline & 6.0800 & 2.51612 & 114 & 5.4112 & 2.34478 & 214 & 4.0665 & 1.16652 \\
\hline & & 115 & 4.7969 & 1.29312 & 215 & 3.9546 & 1.00444 \\
\hline Total & & 116 & 5.8562 & 2.00752 & 216 & 4.6885 & 1.39697 \\
\hline
\end{tabular}

Test for homogeneity of variance was conducted in these data using Levene's test with a probability of 0.056 larger than a significance level of 0.05 , which satisfied the prerequisite for ANOVA. One-way ANOVA was conducted on these 34 sets of data. It is supposed that different types of interface layout have no significant effects on total fixation duration, and the significance level is 0.05 . Results showed that the null hypothesis was rejected if $\mathrm{F}=3.476$ and $\mathrm{P}<0.05$. Thus, in the search task, different types of interface layout had significant effects on total fixation duration.

In conclusion, among layouts Type A, mean total fixation durations of layouts 5 and 9 were significantly shorter than those of layouts 4,7 and 10 , with a significant difference; among layouts Type B1, mean total fixation durations of layouts 101 and 103 were significantly shorter than that of layout 106 , with a significant difference; among layouts Type B2, mean total fixation durations of layouts 111 and 112 were significantly shorter than those of layouts 114 and 116 with a significant difference. However, there was no significant difference among layouts 112, 113 and 115 . By pairwise comparison of all Type $\mathrm{C} 1$ layouts, there was no significant difference; among Type $\mathrm{C} 2$ layouts, the mean total fixation duration of layout 211 was significantly shorter than those of layouts 213 and 216 with a significant difference.

\subsection{Analysis of scanning paths}

An analysis was conducted of scanning paths when subjects performed the experimental task, and the following characteristics were found as follows:

a) Overall fixation points of layouts Type $\mathrm{C}$ were relatively concentrated; layouts $205,213,215$ and 216 showed longer scanning distances and unsmooth paths. The scanning path of layout 215 was illustrated in Figure 8. There was little difference among other layouts Type $\mathrm{C}$ with shorter scanning distances. Figure 9 showed the scanning path of layout 201 , which was a typical scanning path of layout Type C.

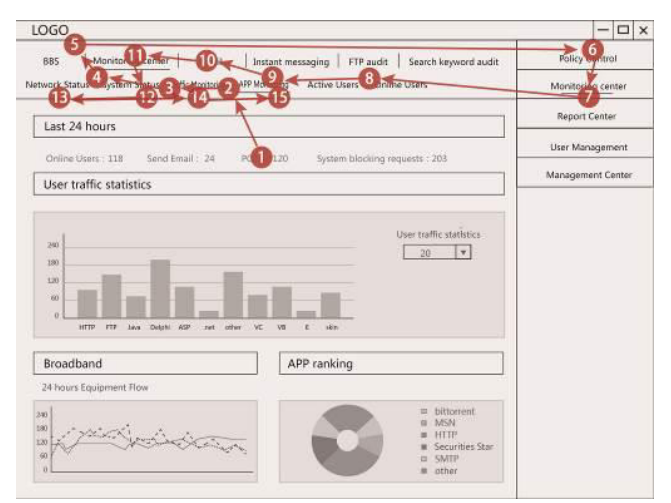

Figure 8. Schematic diagram of scanning path of layout 215

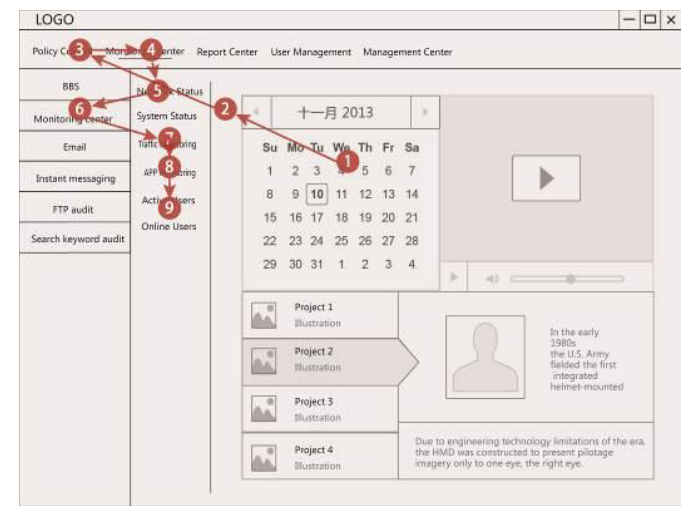

Figure 9. The schematic diagram of scanning path of layout 201

b) In layouts Type A and Type B, not all local points of fixation were scattered; layouts $1,2,5$, 101 and 111 showed relatively clear and smooth scanning paths with less cross points and short scanning paths. The scanning path of layout 1 was illustrated in Figure 10. 


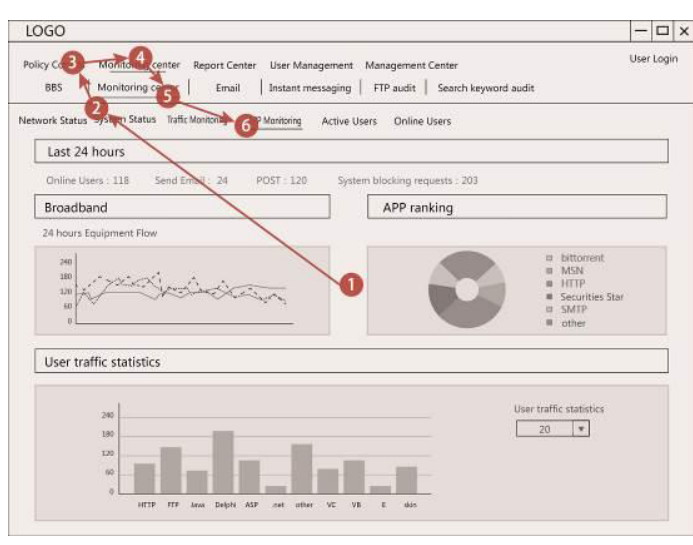

Figure 10. Schematic diagram of scanning path of layout 1

c) From the overall point of view, layouts 6, 7, 104, $105,106,113,114$ and 115 showed obviously longer scanning paths with more cross points and unsmooth lines of sight for subjects. The scanning path of layout 113 was illustrated in Figure 11.

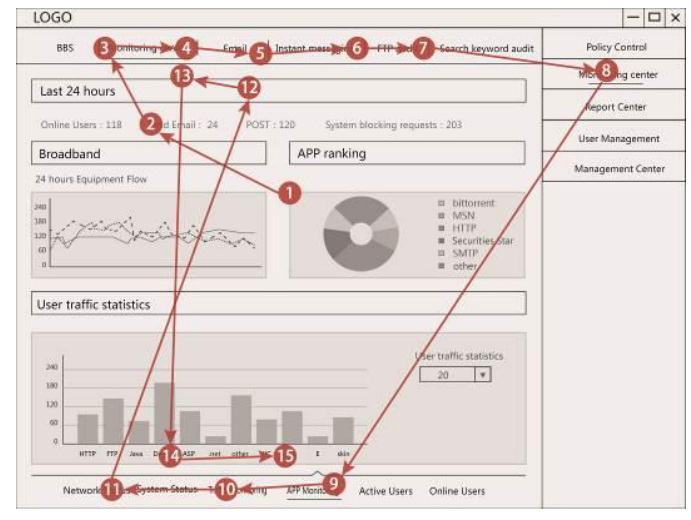

Figure 11. Schematic diagram of scanning path of layout 113

\subsection{Analysis of experimental results}

The results could be seen from data analysis of numbers of fixation points, fixation durations and scanning paths of interfaces that:

a) For multi-task interface, layouts Type $\mathrm{C}$ are superior in whole; no significant difference is noted among the layouts Type $\mathrm{C} 1$ and Type $\mathrm{C} 2$.

b) As a whole, layouts 5, 9, 101, 111, 201, 202, 204, 211, 212 and 214 are superior, whereas layouts $6,7,106,116,205,213,215$ and 216 are inferior.

Meanwhile, it can be concluded that, for either superior or inferior interface, there is certain correlation between layouts on the left and right, and further suggesting that the structures of navigation elements have great influence on subjects' interface information acquisition in the multilevel digital interface, and that introduction becomes more important as task complexity changes. This also verifies the effectiveness of starting with task characteristics in classification of digital interface layouts.

\section{CONCLUSION}

a) According to the task characteristics of digital interfaces for complex systems, layout structures of multilevel digital interfaces can be subdivided into 34 types.

b) Interface elements can be classified as navigation elements and task elements. Based on characteristics of structures and positions of navigation elements, matching relations can be constructed between different types of digital interfaces and layouts by the principle of human visual perception and characteristics of line of sight.

c) Based on the eye tracking technology, advantages and disadvantages of different types of layout are assessed by the searching efficiency of subject-performed task. A matching model which is appropriate for multilevel digital interface layout is constructed by analysis of data and results. Also, the fact that the task element is a significant and important aspect of layout design is verified.

Subdivision of layout types of multilevel digital interfaces provides a scientific experimental model for research on digital interfaces for complex systems, while conclusions of the eye movement experiment provide a reference for layout designs of interfaces for complex systems with different task characteristics. However, influencing factors of interface layouts are highly complex. Therefore, this article presents a design method only based on the structural classification and element characteristics. Designs of color, character and space are also important aspects influencing on interface layout and user's information acquisition. Further research would be made into these influencing factors.

\section{ACKNOWLEDGEMENT}

This paper is sponsored by National Natural Science Foundation of China (No. 71271053, 71471037), Aeronautical Science Foundation of China (No. 20135169016) and Scientific Innovation Research of College Graduates in Jiangsu Province (No. CXLX13082). 


\section{REFERENCES}

[1] Yan Jiaqiong. 2010. Elementary analysis on the layout scheme in network page design. Computer Study, 2: 41-44.

[2] Luo Shijian, Zhu Shangshang. 2010. User Experience and Product Innovation Design. Beijing: China Machine Press, pp: 170-172.

[3] Altaboli, A., \& Lin, Y. 2011. Objective and subjective measures of visual aesthetics of website interface design: the two sides of the coin. In Human-Computer Interaction. Design and Development Approaches. Springer Berlin Heidelberg. pp: 35-44.

[4] Singh, N., \& Bhattacharya, S. (2010, December). A GA-based approach to improve web page aesthetics. In Proceedings of the First International Conference on Intelligent Interactive Technologies and Multimedia. ACM. pp: 29-32.

[5] Teng Hong, Wang Zhengdong, Tu Shandong, et al. 2008. Experimental study on layout design for general interface in process plant for process plants. Machine Design and Research, 24(5): 79-82+87.

[6] Wang Miaohui. 2011. Study on design of visual information interface based on visual cognition. Packaging Engineering, 32(8): 49-51+55.

[7] Zhao Naidi. 2012. Webpage Layout Influence over Visual Search Efficiency with Eye Movement Technology. Shanghai: Fudan University,

[8] Li Ting. 2012. Eye Control Interface Design and Case Development. Hangzhou: Zhejiang University.

[9] Li Leshan. 2004. Human-Computer Interface Design. Beijing: Science Press, pp: 91.

[10]Cheng Shiwei, Sun Zhiqiang. 2014. An approach to eye tracking for mobile device based interaction. Journal of Computer-Aided Design \& Computer Graphics. (8): 1354-1361.

[11]Jin Xiaoping, Qiu Ying, Mao Rongen, et al. 2008. Human-research on driving computer interface layout design reasoning system. Transactions of the Chinese Society for Agricultural Machinery, 39(4): 183-186.

[12] Wang Haiyan, Bian Ting, Xue Chengqi. 2011. Layout design of display interface for a new generation fighter. Electro - Mechanical Engineering, 27(4): 57-61.

[13] Weinreich, H., Obendorf, H., Herder, E., \& Mayer, M. 2008. Not quite the average: An empirical study of Web use. ACM Transactions on the Web (TWEB), 2(1): 5.

[14]Byström, K., Limberg, L., Pejtersen, A. M., Pharo, N., Sundin, O., Belkin, N., \& Kuhlthau, C. 2004. Conceptions of task as a methodological issue: Scandinavians on information seeking and retrieval research (SIG USE). Proceedings of the American Society for Information Science and Technology, 41(1): 577-579.

[15]Williams, M. E. 1979. Annual review of information science and technology.

[16] Campbell, D. J. 1988. Task complexity: A review and analysis. Academy of management review, 13(1): 40-52.

[17]Farzan, R., \& Brusilovsky, P. 2009. Social navigation support for information seeking: If you build it, will they come?. In User Modeling, Adaptation, and Personalization. Springer Berlin Heidelberg. pp: 66-77.
[18]Kammerer, Y., \& Gerjets, P. 2010. How the interface design influences users' spontaneous trustworthiness evaluations of web search results: comparing a list and a grid interface. In Proceedings of the 2010 Symposium on Eye-Tracking Research \& Applications. ACM. pp: 299-306 